\title{
Stability and Convergence of a Finite Element Method for Solving the Stefan Problem ${ }^{1)}$
}

By

Masatake MORI*

\begin{abstract}
A finite element method based on the time dependent basis functions is presented for solving a one phase Stefan problem for the heat equation in one space dimension. The stability and the convergence of the method are studied, and a numerical example is given.
\end{abstract}

\section{$\S 1$. One Phase Stefan Problem in One Space Dimension}

We consider the following one phase Stefan problem in one space dimension. The main equation describing the dynamics of the system is the heat equation

$$
\frac{\partial u}{\partial t}=\sigma \frac{\partial^{2} u}{\partial x^{2}} \quad \text { in } \quad 0<x<s(t), \quad 0<t \leqq T,
$$

associated with a free boundary condition given below. $s(t)$ denotes the position of the free boundary. $\sigma$ is assumed to be a positive constant, and $T$ is an arbitrarily fixed positive number. At the boundary $x=0$ we assume a Dirichlet type boundary condition and at $x=s(t)$ we assume $u=0$ :

$$
\left\{\begin{array}{l}
u(0, t)=g(t) \\
u(s(t), t)=0
\end{array} \text { for } \quad 0 \leqq t \leqq T .\right.
$$

To the initial condition we assign

$$
u(x, 0)=f(x) \geqq 0 \text { for } 0 \leqq x \leqq b, b=s(0)>0 .
$$

Received May 21, 1976.

* Research Institute for Mathematical Sciences, Kyoto University, Kyoto 606, Japan.

1) Part of this work was accomplished while the author was visiting at the Courant Institute of Mathematical Sciences, New York University during 1974-75 academic year. 
The free boundary moves according to the following equation called the Stefan condition:

$$
\frac{d s}{d t}=-\kappa u_{x}(s(t), t) \quad \text { for } \quad 0<t \leqq T,
$$

where $\kappa$ is assumed to be a positive constant. Furthermore for the initial data we make

Assumption A. $0 \leqq f(x) \leqq B(b-x)$,

where $B$ is a positive constant.

It has been shown by Cannon and Hill [2] that the Stefan problem $(1 \cdot 1) \sim(1 \cdot 4)$ has a unique solution under Assumption A. Many approximating methods have been presented for solving the one phase Stefan problem in one dimension numerically. Landau [6] applied a variable transformation in order to change the varying interval $0 \leqq x \leqq s(t)$ into a fixed interval and employed the finite difference method. Douglas and Gallie [3] and Nogi [9] proposed finite difference methods in which an equi-distant space partition is employed and the time variable is discretized in such a way that the free boundary always coincides with a mesh point. Kawarada and Natori [5] combined the penalty method and the finite difference method. Bonnerot and Jamet [1] partitioned the space-time domain into quadrilateral elements and applied the two dimensional finite element method.

In the preceding paper [7] we presented a finite element method (FEM) for the problem $(1 \cdot 1) \sim(1 \cdot 4)$ based on the time dependent basis functions. In the present paper we shall study the stability and the convergence of the method.

\section{$\S 2$. Application of the Finite Element Method and the Scheme}

Consider the domain $0 \leqq x \leqq s(t)$ at time $t$. We partition $0 \leqq x \leqq s(t)$ into $n$ subintervals in accordance with some rule in such a way that the end point of partition always coincides with the free boundary, and denote each node as $x_{j}$ :

$$
0=x_{0}<x_{1}<\cdots \cdots<x_{n}=s(t)
$$


Although $s(t)$ is an unknown function of $t$ which should be determined simultaneously together with $u(x, t)$, we compute $s(t)$ and $u(x, t)$ alternatively in the actual process of computation by means of a similar technique to the idea of "retarding the argument" by Cannon and Hill [2], and hence we write $s_{n}(t)$ instead of $s(t)$ in order to show explicitly that it is an approximation.

We construct piecewise linear basis functions $\left\{\phi_{j}\right\}$ for FEM as shown in Fig. 1:

$$
\phi_{j}(x, t)=\left\{\begin{array}{cl}
\frac{x-x_{j-1}}{x_{j}-x_{j-1}} ; & x_{j-1}<x \leqq x_{j} \\
\frac{x_{j+1}-x}{x_{j+1}-x_{j}} ; & x_{j}<x \leqq x_{j+1} \\
0 \quad ; \text { otherwise. }
\end{array}\right.
$$

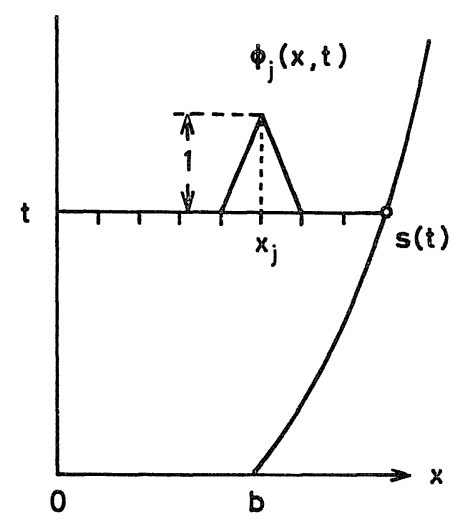

Fig. 1. The basis function $\phi_{j}(x, t)$.

For $\phi_{0}$ and $\phi_{n}$ we take the components of $(2 \cdot 2)$ in $0 \leqq x \leqq x_{1}$ and $x_{n-1}$ $<x \leqq x_{n}$, respectively. $\phi_{j}(x, t)$ depends on time $t$ through $s_{n}(t)$, and its derivatives with respect to $x$ and $t$ are given as

$$
\frac{\partial \phi_{j}}{\partial x}=\left\{\begin{array}{cl}
\frac{1}{x_{j}-x_{j-1}} ; & x_{j-1}<x \leqq x_{j} \\
-\frac{1}{x_{j+1}-x_{j}} ; & x_{j}<x \leqq x_{j+1} \\
0 \quad ; \text { otherwise }
\end{array}\right.
$$


$(2 \cdot 4) \quad \frac{\partial \phi_{j}}{\partial t}=\left\{\begin{array}{cc}-\frac{\left(x-x_{j-1}\right) \dot{x}_{j}+\left(x_{j}-x\right) \dot{x}_{j-1}}{\left(x_{j}-x_{j-1}\right)^{2}} ; x_{j-1}<x \leqq x_{j} \\ \frac{\left(x-\frac{\left.x_{j}\right) \dot{x}_{j+1}+\left(x_{j+1}-x\right) \dot{x}_{j}}{\left(x_{j+1}-x_{j}\right)^{2}}\right.}{0}, x_{j}<x \leqq x_{j+1} \\ 0, \text { otherwise, }\end{array}\right.$

where

$$
\dot{x}_{j}=\frac{d x_{j}}{d t}
$$

Henceforth we partition $0 \leqq x \leqq s_{n}(t)$ into $n$ equal subintervals for simplicity:

$$
x_{j}=j h_{n}(t), \quad h_{n}(t)=\frac{1}{n} s_{n}(t) .
$$

Now we apply the Galerkin method based on the basis functions $\left\{\phi_{j}\right\}$ just constructed above. We expand the approximate solution $u_{n}(x, t)$ of $(1 \cdot 1) \sim(1 \cdot 4)$ in terms of a linear combination of $\phi_{j}(x, t)$ 's:

$$
u_{n}(x, t)=\sum_{j=0}^{n} a_{j}(t) \phi_{j}(x, t)
$$

where

$$
a_{0}(t)=g(t), \quad a_{n}(t)=0
$$

in accordance with the boundary condition $(1 \cdot 2)$. Then we substitute (2.6) into $u$ of (1.1), multiply $\phi_{i}$, and integrate over $0<x<s_{n}(t)$. Then we have a system of ordinary differential equations

$$
M(t) \frac{d \boldsymbol{a}(t)}{d t}=-(\sigma K(t)+N(t)) \boldsymbol{a}(t)
$$

where $\boldsymbol{a}(t)$ is an $(n+1)$-dimensional vector defined by

$$
\boldsymbol{a}(t)=\left(\begin{array}{c}
a_{0}(t) \\
a_{1}(t) \\
\vdots \\
a_{n}(t)
\end{array}\right)
$$

The first and the last elements of (2.9) are known functions of $t$ as seen from $(2 \cdot 7) . \quad M, K$ and $N$ are time dependent $(n-1) \times(n+1)$ matrices, the elements of which are given as follows for $i=1,2, \cdots, n-1$; 
$j=0,1, \cdots, n$ :

$$
\begin{aligned}
M_{i j} & =\int_{0}^{s_{n}(t)} \phi_{i} \phi_{j} d x \quad ; \text { mass matrix } \\
K_{i j} & =\int_{0}^{s_{n}(t)} \frac{\partial \phi_{i}}{\partial x} \times \frac{\partial \phi_{j}}{\partial x} d x ; \text { stiffness matrix } \\
N_{i j} & =\int_{0}^{s_{n}(t)} \phi_{i} \times \frac{\partial \phi_{j}}{\partial t} d x \quad ; \text { velocity matrix. }
\end{aligned}
$$

Since the matrix $N$ corresponds to the apparent velocity of the nodes, we gave it a name "velocity matrix". In usual FEM, we take away the first and the last columns from $M$ and $K$, and call thus obtained square $(n-1) \times(n-1)$ matrices the mass matrix and the stiffness matrix, respectively.

The above definition of $M$ is for the consistent mass system. For the lumped mass system characteristic functions

$$
\psi_{j}(x, t)=\left\{\begin{array}{l}
1 ; \frac{1}{2}\left(x_{j-1}+x_{j}\right)<x \leqq \frac{1}{2}\left(x_{j}+x_{j+1}\right) \\
0 ; \text { otherwise }
\end{array}\right.
$$

are used instead of $\phi_{j}(x, t)$ in the definition of $M$, i.e.

$$
M_{i j}=\int_{0}^{s_{n}(t)} \psi_{i} \psi_{j} d x
$$

The explicit forms of the elements of the matrices are as follows:

Lumped mass system

$$
\begin{aligned}
& M_{i j}=\left\{\begin{array}{cr}
\frac{1}{2}\left(x_{i+1}-x_{i-1}\right)=h_{n} ; & j=i \\
0 \quad ; j \neq i
\end{array}\right. \\
& K_{i j}=\left\{\begin{array}{cl}
-\frac{1}{x_{i}-x_{i-1}}=-\frac{1}{h_{n}} & ; j=i-1 \\
\frac{1}{x_{i}-x_{i-1}}+\frac{1}{x_{i+1}-x_{i}}=\frac{2}{h_{n}} ; j=i & ; j=i+1 \\
-\frac{1}{x_{i+1}-x_{i}}=-\frac{1}{h_{n}} & ; \text { otherwise }
\end{array}\right.
\end{aligned}
$$




$$
N_{i j}=\left\{\begin{array}{cl}
\frac{1}{3} \dot{x}_{i}+\frac{1}{6} \dot{x}_{i-1}=\frac{1}{6}(3 i-1) \frac{d h_{n}}{d t} & ; j=i-1 \\
\frac{1}{6}\left(\dot{x}_{i+1}-\dot{x}_{i-1}\right)=\frac{1}{3} \times \frac{d h_{n}}{d t} & ; j=i \\
-\frac{1}{6} \dot{x}_{i+1}-\frac{1}{3} \dot{x}_{i}=-\frac{1}{6}(3 i+1) \frac{d h_{n}}{d t} ; j=i+1 \\
0 \quad \text {; otherwise. }
\end{array}\right.
$$

\section{Consistent mass system}

In this case only the mass matrix should be changed as follows:

$$
M_{i j}= \begin{cases}\frac{1}{6}\left(x_{i}-x_{i-1}\right)=\frac{1}{6} h_{n} & ; j=i-1 \\ \frac{1}{3}\left(x_{i+1}-x_{i-1}\right)=\frac{2}{3} h_{n} ; j=i \\ \frac{1}{6}\left(x_{i+1}-x_{i}\right)=\frac{1}{6} h_{n} & ; j=i+1 \\ 0 & ; \text { otherwise. }\end{cases}
$$

In the next step we discretize the time variable $t$, i.e. we partition $0 \leqq t \leqq T$ into $m$ equal subintervals with a constant mesh size $\Delta t$ :

$$
\Delta t=\frac{T}{m}, t_{k}=k \Delta t, \quad k=0,1, \cdots, m .
$$

We replace the time derivative of $\boldsymbol{a}(t)$ by the time difference:

$$
\frac{d \boldsymbol{a}(k \Delta t)}{d t} \doteqdot \frac{\boldsymbol{a}(k \Delta t)-\boldsymbol{a}((k-1) \Delta t)}{\Delta t}
$$

and we write

$$
a_{j}^{k}=a_{j}(k \Delta t) .
$$

In this way we have a system of linear equations with respect to $\boldsymbol{a}(k \Delta t)$ corresponding to $(2 \cdot 8)$. If we employ the values at $t=(k-1) \Delta t$ in $\boldsymbol{a}(t)$ of the right hand side of $(2 \cdot 8)$, we have a forward difference scheme. If we employ, on the other hand, the values at $t=k \Delta t$, we have a backward one. In actual computation we can mix them in the ratio $\theta: 1-\theta$ by introducing a parameter $\theta, 0 \leqq \theta \leqq 1$, as will be shown later. 
We compute the increment $\Delta s_{n}$ of $s_{n}(t)$ from $\mathrm{t}=(k-1) \Delta t$ to $t=k \Delta t$ by approximating the right hand side of $(1.4)$ by the gradient of the approximate solution $u_{n}(x, t)$ at the free boundary $x=s_{n}(t)$, and by replacing the left hand side of $(1 \cdot 4)$ by the time difference $\Delta s_{n} / \Delta t$. For the computation of the velocity matrix $N$, we employ an approximation

$$
\frac{d h_{n}}{d t} \fallingdotseq \frac{1}{n} \times \frac{\Delta s_{n}}{\Delta t}
$$

We summarize here the whole scheme obtained in the above procedure. $\theta$ is fixed to a value between 0 and 1 throughout the computation.

\section{Initial routine}

$$
\left\{\begin{array}{l}
a_{j}{ }^{0}=f\left(x_{j}\right), \quad j=0,1, \cdots, n \\
\Delta s_{n}(\Delta t)=\kappa \frac{a_{n-1}^{0}}{h_{n}(0)} \Delta t \\
s_{n}(\Delta t)=b+\Delta s_{n}(\Delta t)
\end{array}\right.
$$

\section{General routine}

Repeat the following process for $k=1,2, \cdots, m$. Compute $M, K$ and $N$ at $t=k \Delta t$ using the values of $s_{n}(k \Delta t)$ and $\Delta s_{n}(k \Delta t)$, and solve the following simultaneous linear equations for $\boldsymbol{a}(k \Delta t)$ :

$$
\begin{aligned}
\{M+ & 0 \Delta t(\sigma K+N)\} \boldsymbol{a}(k \Delta t) \\
& =\{M-(1-\theta) \Delta t(\sigma K+N)\} \boldsymbol{a}((k-1) \Delta t) .
\end{aligned}
$$

Then compute $\Delta s_{n}((k+1) \Delta t)$ and $\left.s_{n}((k+1)\rfloor t\right)$ according to the following equations using the known data $\boldsymbol{a}((k-1)\lrcorner t)$ and $\boldsymbol{a}(k \Delta t)$ :

$$
\begin{aligned}
& \Delta s_{n}((k+1) \Delta t)=\frac{\kappa}{2}\left\{\frac{a_{n-1}^{k-1}}{h_{n}((k-1) \Delta t)}+\frac{a_{n-1}^{k}}{h_{n}(k \Delta t)}\right\} \Delta t \\
& s_{n}((k+1) \Delta t)=s_{n}(k \Delta t)+\Delta s_{n}((k+1) \Delta t) .
\end{aligned}
$$

The reason why we adopted the mean value of the data at $t=(k-1)$ $\cdot \Delta t$ and $k \Delta t$ in $(2 \cdot 25)$ is in order to make $s_{n}(t) \in C^{1}$ as seen later (see $(4 \cdot 9))$. 


\section{§3. Stability}

In this section we discuss the stability of the scheme given in $\S 2$. For simplicity we use the following notations:

$$
\begin{aligned}
\alpha_{k} & \equiv \frac{\sigma n^{2} \Delta t}{s_{n}{ }^{2}(k \Delta t)}+\frac{\Delta s_{n}(k \Delta t)}{6 s_{n}(k \Delta t)} \\
\beta_{k} & \equiv \frac{\Delta s_{n}(k \Delta t)}{2 s_{n}(k \Delta t)}
\end{aligned}
$$

For the moment we confine ourselves to the case of the lumped mass system. The stability of the scheme of the consistent mass system will be referred to at the end of this section.

The lumped mass scheme can be explicitly written as follows:

$$
\begin{aligned}
-\theta\left(\alpha_{k}-i \beta_{k}\right) a_{j-1}^{k}+\left\{1+2 \theta \alpha_{k}\right\} a_{j}^{k}-\theta\left(\alpha_{k}+j \beta_{k}\right) a_{j+1}^{k} \\
=(1-\theta)\left(\alpha_{k}-j \beta_{k}\right) a_{j-1}^{k-1}+\left\{1-2(1-\theta) \alpha_{k}\right\} a_{j}^{k-1} \\
\quad+(1-\theta)\left(\alpha_{k}+j \beta_{k}\right) a_{j+1}^{k-1}, \\
j=1,2, \cdots, n-1 ; k=1,2, \cdots, m,
\end{aligned}
$$

where $a_{0}{ }^{k}=g(k \Delta t)$ and $a_{n}{ }^{k}=0$ are known. For the later convenience we introduce the following operator $P_{L}$ :

$$
\begin{aligned}
P_{L}\left(k, j ; \Delta t, s_{n}(k \Delta t), \Delta s_{n}(k \Delta t) ; \theta\right) w_{j}^{k}=P_{L}(k, j) w_{j}^{k} \\
\equiv-\theta\left(\alpha_{k}-j \beta_{k}\right) w_{j-1}^{k}+\left\{1+2 \theta \alpha_{k}\right\} w_{j}^{k}-\theta\left(\alpha_{k}+j \beta_{k}\right) w_{j+1}^{k} \\
\quad-(1-\theta)\left(\alpha_{k}-j \beta_{k}\right) w_{j-1}^{k-1}-\left\{1-2(1-\theta) \alpha_{k}\right\} w_{j}^{k-1} \\
\quad-(1-\theta)\left(\alpha_{k}+j \beta_{k}\right) w_{j+1}^{k-1} .
\end{aligned}
$$

Evidently the scheme $(3 \cdot 3)$ is written as $P_{L}(k, j) a_{j}{ }^{k}=0$.

Lemma $\mathbb{1}$ (Lumped mass system). If

$$
n\left|\beta_{k}\right| \leqq \alpha_{k} \leqq \frac{1}{2(1-\theta)}, \quad 0 \leqq p_{j}{ }^{k}, j=0,1, \cdots, n-1 ; k=1,2, \cdots, m,
$$

then the scheme

$$
P_{L}(k, j) w_{j}^{k}=p_{j}{ }^{k}, j=1,2, \cdots, n-1
$$

satisfies the following maximum principle locally at each $k=1,2, \cdots$, 
$m:$

$$
\begin{array}{r}
\min \left(w_{0}^{k}, w_{n}{ }^{k}, w_{\min }^{k-1}\right) \leqq w_{j}{ }^{k} \leqq \max \left(w_{0}{ }^{k}, w_{n}{ }^{k}, w_{\max }^{k-1}\right)+p_{j}{ }^{k}, \\
j=0,1, \cdots, n,
\end{array}
$$

where

$$
w_{\min }^{k-1}=\min _{0 \leqq j \leqq n} w_{j}^{k-1} \text { and } \quad w_{\max }^{k-1}=\max _{0 \leqq j \leqq n} w_{j}^{k-1}
$$

Proof. The left inequality in (3.7) is trivial if $w_{j}^{k}$ attains the minimum at $j=0$ or at $j=n$. Suppose $w_{j}^{k}$ attains the minimum at $j=M$ $(M \neq 0, n)$. From (3.5) it is evident that $\alpha_{k} \pm j\left|\beta_{k}\right|>0$ and $1-2(1$ $-\theta) \alpha_{k} \geqq 0$, so that we have

$$
\begin{aligned}
& \left\{1+2 \theta \alpha_{k}\right\} w_{M}^{k}=\theta\left(\alpha_{k}-j \beta_{k}\right) w_{M-1}^{k}+\theta\left(\alpha_{k}+j \beta_{k}\right) w_{M+1}^{k} \\
& \quad+(1-\theta)\left(\alpha_{k}-j \beta_{k}\right) w_{M-1}^{k-1}+\left\{1-2(1-\theta) \alpha_{k}\right\} w_{M}^{k-1} \\
& \quad+(1-\theta)\left(\alpha_{k}+j \beta_{k}\right) w_{M+1}^{k-1}+p_{j}{ }^{k} \geqq 2 \theta \alpha_{k} w_{M}{ }^{k}+w_{\min }^{k-1} .
\end{aligned}
$$

Hence the left inequality in $(3 \cdot 7)$ is valid.

Similarly, the right inequality in (3.7) is trivial if $w_{j}^{k}$ attains the maximum at $j=0$ or at $j=n$. Suppose $w_{j}{ }^{k}$ attains the maximum at $j=M^{\prime}$ $\left(M^{\prime} \neq 0, n\right)$. Then we have

$$
\left\{1+2 \theta \alpha_{k}\right\} w_{M^{\prime}}{ }^{k} \leqq 2 \theta \alpha_{k} w_{M^{\prime}}{ }^{k}+w_{\max }^{k-1}+p_{j}{ }^{k},
$$

and so the right inequality in $(3 \cdot 7)$ also holds.

Q.E.D.

Now we introduce the following quantities:

$$
\begin{aligned}
A & \equiv \max \left(\frac{1}{b} \max _{0 \leqq t \leqq T} g(t), B\right) \\
l & \equiv b+\kappa A T \\
\lambda_{b} & \equiv \frac{\sigma \Delta t}{h_{n}^{2}(0)}=\frac{\sigma n^{2} \Delta t}{b^{2}} \\
\lambda_{l} & \equiv \frac{\sigma n^{2} \Delta t}{l^{2}}
\end{aligned}
$$

In addition to Assumption $\mathrm{A}$, we make the following ones: 
Assumption $\mathbf{B}_{\circ} \quad \lambda_{b}\left(1+\frac{\kappa b A}{6 \sigma n^{2}}\right) \leqq \frac{1}{2(1-\theta)}$

Assumption $\mathbb{C}_{\cdot}\left\{\begin{array}{l}A \leqq \frac{2 \sigma n}{\kappa l} \\ A \leqq \frac{b}{(1-\theta) \kappa \Delta t}\end{array}\right.$

Lemma 2 (lumped mass system). Under Assumptions A, B and $C$, we have

$(3 \cdot 13) \quad 0 \leqq \frac{a_{j}{ }^{k}}{\left(1-\frac{j}{n}\right) s_{n}(k \Delta t)} \leqq A ; j=0,1, \cdots, n-1 ; k=0,1, \cdots, m$

Proof. By definition we have

$$
P_{L}(k, j) a_{j}^{k}=0
$$

We define (see Fig. 2)

$$
d_{j}{ }^{k} \equiv A\left(1-\frac{j}{n}\right) s_{n}(k \Delta t)-a_{j}{ }^{k} .
$$

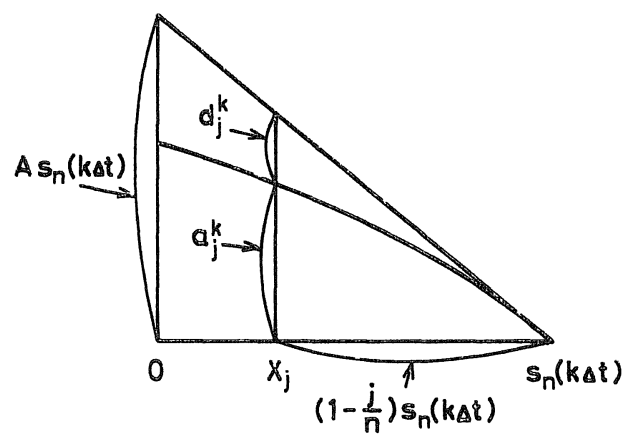

Fig. 2.

It is easy to see that $d_{j}{ }^{k}$ satisfies

$$
P_{L}(k, j) d_{j}{ }^{k}=p_{j}{ }^{k},
$$

where 


$$
p_{j}{ }^{k}=A \Delta s_{n}(k \Delta t)\left\{1-(1-\theta) \frac{j}{n} \times \frac{\Delta s_{n}(k \Delta t)}{s_{n}(k \Delta t)}\right\} .
$$

In place of $(3 \cdot 13)$ we shall prove

$$
\left.\begin{array}{l}
0<b \leqq s_{n}(\Delta t) \leqq s_{n}(2 \Delta t) \leqq \cdots \leqq s_{n}(k \Delta t) \\
0 \leqq a_{j}{ }^{k}, \quad 0 \leqq d_{j}{ }^{k}, \quad j=0,1, \cdots, n-1
\end{array}\right\}
$$

by induction with respect to $k$. It would be clear that $(3 \cdot 18)$ and $(3 \cdot 19)$ imply $(3 \cdot 13)$.

For $k=0,(3 \cdot 18)$ and $(3 \cdot 19)$ are trivial because of $b=s_{n}(0)>0$, (1.3) and Assumption A.

Suppose that (3.18) and (3.19) hold for $0,1, \cdots, k-1$. Since $\left.s_{n}(k\lrcorner t\right) \geqq 0$ from $(2 \cdot 23)$ and $(2 \cdot 25),(3 \cdot 18)$ follows from $(2 \cdot 26)$. Now we claim that

$$
\frac{\Delta s_{n}(k \Delta t)}{\Delta t} \leqq \kappa A
$$

When $k=1$, this is evident from $(2 \cdot 23)$. When $k \geqq 2$, we put $j=n-1$ in (3.13) which is assumed to hold for $k-2$ and $k-1$. Then $(3 \cdot 20)$ follows from $(2 \cdot 25)$. Hence we have

$$
p_{j}{ }^{k} \geqq A \Delta s_{n}(k \Delta t)\left\{1-(1-\theta) \frac{\kappa A}{b} \Delta t\right\} \geqq 0
$$

by the second inequality of Assumption $C$. Here we apply Lemma 1 to $(3 \cdot 14)$ and $(3 \cdot 16)$. What is left to be done is to show $n \beta_{k}<\alpha_{k} \leqq \frac{1}{2(1-0)}$. Note that $s_{n}(k \Delta t) \leqq b+\kappa A k \Delta t \leqq l$ from $(3 \cdot 20)$. Then

$$
\begin{aligned}
\alpha_{k}-n \beta_{k} & =\frac{\Delta t}{s_{n}(k \Delta t)}\left\{\frac{\sigma n^{2}}{s_{n}(k \Delta t)}-\frac{3 n-1}{6} \times \frac{\Delta s_{n}(k \Delta t)}{\Delta t}\right\} \\
& \geqq \frac{\kappa n \Delta t}{2 l}\left(\frac{2 \sigma n}{\kappa l}-A\right) \geqq 0
\end{aligned}
$$

follows from the first inequality of Assumption C. Furthermore from $(3 \cdot 20)$ and Assumption $B$ we have

$$
\frac{1}{2(1-\bar{\theta})}-\alpha_{k} \geqq \frac{\kappa \Delta t}{6 b}\left[\frac{6 \sigma n^{2}}{\kappa b}\left\{\frac{1}{2(1-\theta) \lambda_{b}}-1\right\}-A\right] \geqq 0 .
$$

All the inequalities appearing in the assumptions of Lemma 1 are guaranteed by $(3 \cdot 21),(3 \cdot 22)$ and $(3 \cdot 23)$, so that we can apply it. 
First note that $a_{0}{ }^{k}=g(k \Delta t) \geqq 0, a_{n}{ }^{k}=0$, and that $a_{j}{ }^{k-1} \geqq 0$ by the assumption of induction. Then apply Lemma 1 to $(3 \cdot 14)$, so that we have $a_{j}^{k} \geqq 0$, $j=1,2, \cdots, n-1$. Next note that

$$
d_{0}{ }^{k}=A s_{n}(k \Delta t)-a_{0}{ }^{k} \geqq A b-g(k \Delta t) \geqq 0, d_{n}{ }^{k}=0,
$$

and that $d_{j}{ }^{k-1} \geq 0$ by the assumption of induction. Then the application of Lemma 1 results in $d_{j}{ }^{k} \geqq 0, j=1,2, \cdots, n-1$ by $(3 \cdot 21)$, verifying $(3 \cdot 19)$ with $k$ increased one.

Q.E.D.

Lemma 2 says that under Assumptions $A, B$ and $C$ the assumption (3.5) of Lemma 1 is satisfied. Therefore we conclude that at each time step our scheme satisfies the maximum principle in the same sense as in Lemma 1. Hence for stability we have

Theorem $\mathbb{1}$ (Lumped mass system). Under Assumptions A, B and $C$, the scheme

$$
P_{L}(k, j) a_{j}{ }^{k}=0, j=1,2, \cdots, n-1
$$

is stable in the sense that at each time step $k=1,2, \cdots, m(3 \cdot 24)$ satisfies the following maximum principle:

$$
0 \leqq a_{j}{ }^{k} \leqq \max \left(a_{0}{ }^{k}, a_{\max }^{k-1}\right), j=1,2, \cdots, n-1 .
$$

In the similar way we can show that Theorem 1 holds for the scheme of the consistent mass system

$$
\begin{gathered}
\left\{1-6 \theta\left(\alpha_{k}-j \beta_{k}\right)\right\} a_{j-1}^{k}+\left\{4+12 \theta \alpha_{k}\right\} a_{j}^{k}+\left\{1-6 \theta\left(\alpha_{k}+j \beta_{k}\right)\right\} a_{j+1}^{k} \\
=\left\{1+6(1-\theta)\left(\alpha_{k}-j \beta_{k}\right)\right\} a_{j-1}^{k-1}+\left\{4-12(1-\theta) \alpha_{k}\right\} a_{j}^{k-1} \\
+\left\{1+6(1-\theta)\left(\alpha_{k}+j \beta_{k}\right)\right\} a_{j+1}^{k-1}, \\
j=1,2, \cdots, n-1 ; k=1,2, \cdots, m,
\end{gathered}
$$

if we replace Assumptions $B$ and $C$ by the following ones:

Assumption $\mathbb{B}_{0}\left\{\begin{array}{l}\lambda_{b}\left(1+\frac{\kappa b A}{6 \sigma n^{2}}\right) \leqq \frac{1}{3(1-\theta)} \\ \frac{1}{6 \theta} \leqq \lambda_{l}\left(1-\frac{\kappa l A}{2 \sigma n}\right)\end{array}\right.$ 


\section{Assumption $\mathbb{C}_{\text {。 }} \quad A \leqq \frac{b}{(1-\theta) \kappa \Delta t}$.}

Since $\lambda_{l}<\lambda_{b}, \theta$ must satisfy $1 / 3<\theta$.

Assumption $B$ is always essential both in the lumped and the consistent mass system, while Assumption $C$ becomes trivial as $\Delta t \rightarrow 0$ and $n \rightarrow \infty$. Note that Assumption $B$ corresponds to the stability condition given by Fujii [4] in FEM for the normal heat equation with a fixed boundary.

\section{§ 4. Convergence}

This section is concerned with the convergence of the scheme, i.e. we shall show here that $u_{n}(x, t)$ of $(2 \cdot 6)$ converges to the solution of $(1 \cdot 1) \sim(1 \cdot 4)$ as $\Delta t \rightarrow 0$ and $n \rightarrow \infty$. Henceforth we shall confine ourselves to the lumped mass system with $\theta=1$, i.e. to the scheme

$$
-\left(\alpha_{k}-j \beta_{k}\right) a_{j-1}^{k}+\left(1+2 \alpha_{k}\right) a_{j}^{k}-\left(\alpha_{k}+j \beta_{k}\right) a_{j+1}^{k}=a_{j}^{k-1} .
$$

First we assume that the limit $\Delta t \rightarrow 0$ or $n \rightarrow \infty$ is taken under the following constraint condition:

Assumption D. $\lambda_{b}=\frac{\sigma n^{2} \Delta t}{b^{2}}=$ constant.

Furthermore for the initial and the boundary data we make

Assumption $\mathbb{E}_{\bullet} \quad\left\{\begin{array}{l}f(x) \in C^{2}(x), \quad g(t) \in C^{1}(t) \\ f(0)=g(0), \frac{d g}{d t}(0)=\sigma \frac{d^{2} f}{d x^{2}}(0) .\end{array}\right.$

If we put $j=n-1$ in $(3 \cdot 13)$ we have

$$
0 \leqq \frac{a_{n-1}^{k}}{h_{n}(k \Delta t)} \leqq A
$$

From this inequality, $(3 \cdot 20)$ and Assumption $D$, we have estimates for $\alpha_{k}$ and $\beta_{k}$ : 


$$
\beta_{k} \leqq \frac{\kappa A}{2 b} \Delta t
$$

$$
n \beta_{k} \leqq \frac{\kappa A \sqrt{\lambda_{b}}}{2 \sqrt{\sigma}} \Delta t^{1 / 2}
$$

$$
\frac{b^{2}}{l^{2}} \lambda_{b} \leqq \alpha_{k} \leqq \lambda_{b}+\frac{\kappa A}{6 b} \Delta t
$$

We extend the approximate solution $u_{n}(x, t)$, which is given only at the discrete points $t=k \Delta t$, to that given also at intermediate values of $t$, i.e. at $(k-1) \Delta t<t \leqq k \Delta t$, by interpolating the gradient of $u_{n}(x, t)$ in the following fashion. First we define $z_{n}(t)$ which corresponds to the gradient of $u_{n}(x, t)$ at $x=s_{n}(t)$ by linear interpolation:

$$
\begin{gathered}
\text { (4.6) } z_{n}(t)=\kappa \frac{a_{n-1}^{k-1}}{h_{n}((k-1) \Delta t)}+\kappa\left\{\frac{a_{n-1}^{k}}{h_{n}(k \Delta t)}-\frac{a_{n-1}^{k-1}}{h_{n}((k-1) \Delta t)}\right\} \frac{t-(k-1) \Delta t}{\Delta t}, \\
(k-1) \Delta t<t \leqq k \Delta t, k=1,2, \cdots, m .
\end{gathered}
$$

Next we define $s_{n}(t)$ at $(k-1) \Delta t<t \leqq k \Delta t$ based on the similar idea to that of retarding the argument [2], i.e.

$$
s_{n}(t)=\left\{\begin{aligned}
b+\kappa \frac{a_{n-1}^{0}}{h_{n}(0)} t ; & 0 \leqq t \leqq \Delta t \\
s_{n}((k-1) \Delta t) & +\int_{(k-1) \Delta t}^{t} z_{n}(\tau-\Delta t) d \tau \\
& ;(k-1) \Delta t<t \leqq k \Delta t, k=2,3, \cdots, m .
\end{aligned}\right.
$$

If we put $t=k \Delta t$, we have

$$
\int_{(k-1) \Delta t}^{k \Delta t} z_{n}(\tau-\Delta t) d \tau=\Delta s_{n}(k \Delta t), \quad k=2,3, \cdots, m
$$

which is consistent with $(2 \cdot 25)$. It is clear from this definition that $s_{n}(t)$ is differentiable. In addition to that, the derivative of $s_{n}(t)$

$$
\frac{d s_{n}}{d t}(t)=\left\{\begin{array}{l}
\kappa \frac{a_{n-1}^{0}}{h_{n}(0)}, \quad 0 \leqq t \leqq \Delta t \\
z_{n}(t-\Delta t), \quad \Delta t<t \leqq T
\end{array}\right.
$$

is continuous on $0 \leqq t \leqq T$ because of the continuity of $z_{n}(t)$. Since $s_{n}(t)$ is defined at every $t$, we can construct the basis functions $\phi_{j}(x, t)$ for any $t$ by dividing the interval $0 \leqq x \leqq s_{n}(t)$ into $n$ equal subintervals. 
By linear interpolation of the gradient of $u_{n}(x, t)$ in each interval $(j-1)$ $\times h_{n}(t)<x \leqq j h_{n}(t)$ based on the values at $t=(k-1) \Delta t$ and $k \Delta t$, we have the extended solution $u_{n}(x, t)$ for any value of $x$ and $t$.

By the definition $(4 \cdot 6)$ and from $(4 \cdot 2)$ we have

Lemma 3 (lumped and consistent mass system). Under Assumptions $A, B$ and $C$,

$$
0 \leqq \frac{d s_{n}(t)}{d t} \leqq \kappa A
$$

From this Lemma we see that $\left\{s_{n}(t)\right\}$ is uniformly bounded on $0 \leqq t \leqq T$, i.e.

$$
b \leqq s_{n}(t) \leqq b+\kappa A T=l \text { for } 0 \leqq t \leqq T
$$

and equi-continuous, so that we can select a subsequence from $\left\{s_{n}(t)\right\}$ that converges, i.e., if we write this subsequence as $\left\{s_{n}(t)\right\}$ again, we have for any $\varepsilon>0$

$$
\left|s_{n}(t)-s_{\infty}(t)\right|<\varepsilon,
$$

where $s_{\infty}(t)$ is a limit function.

We consider next a solution $u(x, t)$ of the heat equation $(1 \cdot 1) \sim$ $(1 \cdot 3)$ in which the boundary $s(t)$ is supposed to be given and fixed as $s_{\infty}(t)$. The present purpose is to show that $u_{n}(x, t)$ converges to $u(x, t)$ uniformly as $\Delta t \rightarrow 0 \quad(n \rightarrow \infty)$ in $0<t \leqq T, 0 \leqq x<s_{\infty}(t)$. For that object we introduce an auxiliary function $v_{n}(x, t)$ which is a solution of $(1 \cdot 1) \sim$ $(1 \cdot 3)$ having a fixed boundary $s_{n}(t)$ instead of $s_{\infty}(t)$. Note that $v_{n}(x, t)$ and $u(x, t)$ exist because $s_{n}(t)$ and $s_{\infty}(t)$ are uniformly Lipschitz continuous functions as seen from $(4 \cdot 10)$ (see e.g. [10]).

We compare first $u(x, t)$ with $v_{n}(x, t)$, and secondly $v_{n}(x, t)$ with $u_{n}(x, t)$. For the first step we use

Lemma 4t (Cannon and Hill [2]). Let $s(t)$ be a monotonic nondecreasing function, and $u(x, t)$ be a solution of $(1 \cdot 1) \sim(1 \cdot 3)$. Then under Assumption A

$$
0 \leqq \rho^{-1} u(s(t)-\rho, t) \leqq A
$$


for all $0 \leqq t \leqq T, 0<\rho<b$.

Lemma 2 is nothing but a FEM version of this Lemma 4 .

We extend $u$ and $v_{n}$ in such a way that they are identically equal to zero outside the boundaries $s_{\infty}(t)$ and $s_{n}(t)$, respectively. Note that $u$ and $v_{n}$ have common initial and boundary data. For any $\varepsilon>0$ let $\rho=\varepsilon / A$. For sufficiently large $n$ we have

$$
\left|s_{n}(t)-s_{\infty}(t)\right| \leqq \rho
$$

from (4-12). Then using Lemma 4 and from the maximum principle [2], we have

$$
\left|v_{n}(x, t)-u(x, t)\right| \leqq A \rho=\varepsilon
$$

in $0 \leqq x \leqq \max \left(s_{n}(t), s_{\infty}(t)\right), 0 \leqq t \leqq T$, which shows the convergence of $v_{n}$ to $u$.

In the second step we compare $v_{n}(x, t)$ with $u_{n}(x, t)$. In order to prove that $\left|u_{n}(x, t)-v_{n}(x, t)\right|<\varepsilon$ in $0 \leqq x<s_{\infty}(t), 0<t \leqq T$ for sufficiently large $n$, we shall show it in the domain

$$
D_{\delta} \equiv\left\{(x, t) \mid 0 \leqq x \leqq s_{\infty}(t)-\delta, 0 \leqq t \leqq T\right\}
$$

for any arbitrarily small $\delta>0$. We define

$$
\delta^{\prime}=\min \left(\delta, \frac{\varepsilon}{A}\right),
$$

and prove that $\left|u_{n}-v_{n}\right|<\varepsilon$ in $D_{\hat{o}^{\prime}}$ since the inequality $\left|u_{n}-v_{n}\right|<\varepsilon$ in $D_{\delta^{\prime}}$ implies that in $D_{\delta}$. Let $n_{0}$ be a sufficiently large integer. Then there exist such $\delta_{1}, 0<\delta_{1}<\delta^{\prime}$, and $J<n$ for any $n>n_{0}$ that

$$
\begin{gathered}
s_{\infty}(k \Delta t)-\delta^{\prime}<x_{J}^{k}<s_{\infty}(k \Delta t)-\delta_{1}, \quad x_{J}^{k}=\frac{J}{n} s_{n}(k \Delta t) \\
s_{n}(k \Delta t)-\delta^{\prime}<x_{J}^{k}<s_{n}(k \Delta t)-\delta_{1},
\end{gathered}
$$

for all $k=0,1, \cdots, m$ (see Fig. 3). What we plan to do is to show that for sufficiently large $n$

$$
\left|u_{n}\left(x_{j}{ }^{k}, k \Delta t\right)-v_{n}\left(x_{j}^{k}, k \Delta t\right)\right|<\varepsilon
$$

for $x_{j}{ }^{k}=\frac{j}{n} s_{n}(k \Delta t) ; j=0,1, \cdots, J ; k=1,2, \cdots, m . \quad$ Note that $\frac{\partial v_{n}}{\partial t}, \frac{\partial^{2} v_{n}}{\partial x^{2}}$ and 


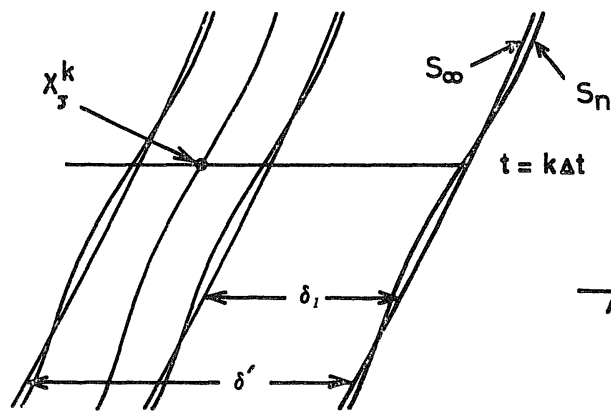

Fig. 3.

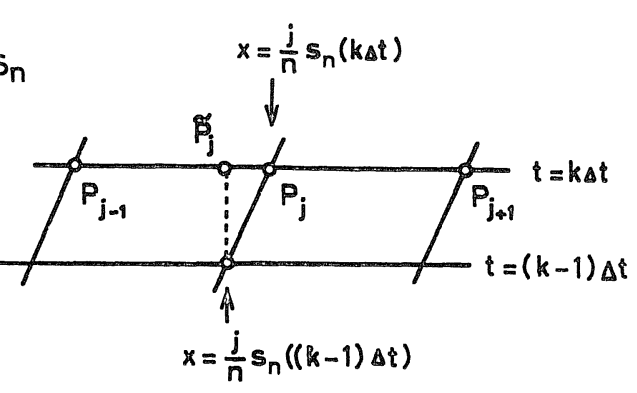

Fig. 4.

$\frac{\partial u}{\partial t}, \frac{\partial^{2} u}{\partial x^{2}}$, where $u$ is the limit function of $v_{n}$, are uniformly continuous in $D_{\delta_{1}}$ if we fix $\delta_{1}$ (see e.g. [10]).

Let $v_{j}{ }^{k}$ be the value of $v_{n}$ at the node $P_{j}\left(x=\frac{j}{n} s_{n}(k \Delta t), t=k \Delta t\right)$, i.e.

$$
v_{j}^{k} \equiv v_{n}\left(x_{j}^{k}, k \Delta t\right)
$$

and define the difference

$$
\varepsilon_{j}^{k} \equiv a_{j}^{k}-v_{j}^{k} .
$$

Since the initial and the boundary data are common, we have

$$
\varepsilon_{0}^{k}=0, \quad \varepsilon_{j}^{0}=0 \text {. }
$$

For the difference in the neighborhood of the free boundary we use Lemmas 2 and 4, and obtain an estimate

$$
\left|\varepsilon_{J}{ }^{k}\right| \leqq \max \left(u_{n}\left(x_{J}^{k}, k \Delta t\right), v_{n}\left(x_{J}^{k}, k \Delta t\right)\right)<\varepsilon
$$

from $(4 \cdot 16)$ and $(4 \cdot 18)$.

It is easy to check that $\varepsilon_{j}^{k}$ satisfies

$$
P_{L}\left(k, j ; \Delta t, s_{n}, \Delta s_{n} ; 1\right) \varepsilon_{j}{ }^{k}=-P_{L}\left(k, j ; \Delta \iota, s_{n}, \Delta s_{n} ; 1\right) v_{j}{ }^{k} .
$$

We denote the point $x=\frac{j}{n} s_{n}((k-1) \Delta t), t=k \Delta t$ as $\widetilde{P}_{j}$ as is shown in Fig. 4, and put

$$
\widehat{v}_{j}^{k} \equiv j \frac{\Delta s_{n}}{2 s_{n}} v_{j-1}^{k}+v_{j}^{k}-j \frac{\Delta s_{n}}{2 s_{n}} v_{j+1}^{k}
$$

as an approximate value of $v_{j}{ }^{k} . \quad \widetilde{v}_{j}{ }^{k}$ is the mean value of the linear interpolation of $v_{n}(x, t)$ based on the abscissas $P_{j-1}$ and $P_{j}$ and that based 
on $P_{j}$ and $P_{j+1}$ in Fig. 4. The difference between $v_{n}$ at $\widetilde{P}_{j}$ and $\widetilde{v}_{j}{ }^{k}$ is given as

$$
\begin{aligned}
v_{n}\left(\widetilde{P}_{j}\right)-\widetilde{v}_{j}{ }^{k}= & \frac{1}{2}\left\{\frac{1}{2 !}\left(\frac{j}{n} \Delta s_{n}\right)\left(\frac{s_{n}-\Delta s_{n}}{n}\right) \frac{\overline{\partial^{2} u}}{\partial x^{2}}\right. \\
& \left.+\frac{1}{2 !}\left(\frac{j}{n} \Delta s_{n}\right)\left(\frac{s_{n}+\Delta s_{n}}{n}\right) \frac{\overline{\partial^{2} u}}{\partial x^{2}}\right\}
\end{aligned}
$$

by the error formula for the Lagrange interpolation, where $\frac{\overline{\partial^{2}} \bar{u}}{\partial x^{2}}$ and $\frac{\overline{\partial^{2} u}}{\partial x^{2}}$ are values of $\frac{\partial^{2} u}{\partial x^{2}}$ at certain points in the interval of interpolation and are uniformly bounded in $D_{\delta_{1}}$. On the other hand, since $\Delta s_{n}=O(\Delta t)$ by $(3 \cdot 2)$ and $(4 \cdot 3)$, we have for any $\varepsilon>0$

$$
\left|v_{n}\left(\widetilde{P}_{j}\right)-\widetilde{v}_{j}{ }^{k}\right|<\varepsilon \Delta t
$$

for sufficiently large $n$. Furthermore, if we write the right hand side of $(4 \cdot 24)$ explicitly, we have

$$
\begin{aligned}
& -P_{L}\left(k, j ; \Delta t, s_{n}, \Delta s_{n} ; 1\right) \varepsilon_{j}{ }^{k} \\
& \quad=\Delta t\left\{\frac{\widetilde{v}_{j}{ }^{k}-v_{j}{ }^{k-1}}{\Delta t}-\sigma\left(1+\frac{s_{n}}{6 \sigma n^{2}} \times \frac{\Delta s_{n}}{\Delta t}\right) \frac{v_{j-1}^{k}-2 v_{j}{ }^{k}+v_{j+1}^{k}}{h_{n}{ }^{2}(k \Delta t)}\right\} .
\end{aligned}
$$

Note that $\frac{\partial v_{n}}{\partial t}$ and $\frac{\partial^{2} v_{n}}{\partial x^{2}}$ are uniformly continuous in $D_{\delta_{1}}$, so that for sufficiently large $n$ we have

$$
\left|\frac{\partial v_{n}}{\partial t}\left(\widetilde{P}_{j}\right)-\frac{v_{n}\left(\widetilde{P}_{j}\right)-v_{j}^{k-1}}{\Delta t}\right|<\varepsilon
$$

and

$$
\left|\frac{\partial^{2} v_{n}}{\partial x^{2}}\left(\hat{P}_{j}\right)-\frac{v_{j-1}^{k}-2 v_{j}^{k}+v_{j+1}^{k}}{h_{n}^{2}(k \Delta t)}\right|<\varepsilon
$$

for any $\varepsilon>0$. Since $v_{n}$ satisfies $(1 \cdot 1)$, we have from $(4 \cdot 27)$

$$
\left|P_{L}\left(k, j ; \Delta t, s_{n}, \Delta s_{n} ; 1\right) \varepsilon_{j}{ }^{k}\right|<\varepsilon \Delta t
$$

for sufficiently large $n$. Therefore by Lemma 1 together with (4.22) and $(4 \cdot 23)$ we have

$$
\left|\varepsilon_{j}{ }^{k}\right|=\left|v_{n}\left(x_{j}^{k}, \Delta t\right)-u_{n}\left(x_{j}^{k}, \Delta t\right)\right|<\varepsilon, j=0,1, \cdots, J,
$$

showing that $\left|u_{n}(x, t)-v_{n}(x, t)\right|<\varepsilon$ in $0 \leqq x<s_{\infty}(t), 0<t \leqq T$ as $\Delta t \rightarrow 0$ 
$(n \rightarrow \infty)$.

From $(4 \cdot 14)$ and $(4 \cdot 30)$ we conclude that the approximate solution $u_{n}(x, t)$ converges to $u(x, t)$ in $0 \leqq x<s_{\infty}(t), 0<t \leqq T$ as $\Delta t \rightarrow 0(n \rightarrow \infty)$.

What is left to be proved is that $u(x, t)$, which is the solution of $(1 \cdot 1) \sim(1 \cdot 3)$ with the boundary $s_{\infty}(t)$, satisfies the Stefan condition $(1 \cdot 4)$. For that purpose we define the second difference of $a_{i}{ }^{k}$ :

$$
c_{j}^{k}=\frac{a_{j-1}^{k}-2 a_{j}^{k}+a_{j+1}^{k}}{h_{n}^{2}(k \Delta t)}, \quad j=1,2, \cdots, n-1 .
$$

We need the following two lemmas.

Lemma 5 (lumped mass system with $\theta=1$ ). Under Assumptions $A, B, C$ and $E,\left\{c_{j}{ }^{k}\right\}$ is uniformly bounded, i.e.

$$
\left|c_{i}{ }^{k}\right| \leqq M, j=1,2, \cdots, n-1 ; k=1,2, \cdots, m \text {. }
$$

Proof. We extend the definition of the scheme (4.1), which was originally defined only for $1 \leqq j \leqq n-1$, to $j=0$ and $n$, and define $a_{-1}^{k}$ and $a_{n+1}^{k}$ consistently. By this extension $c_{0}{ }^{k}$ and $c_{n}{ }^{k}$ can also be defined.

Putting $j=0$ in $(4 \cdot 1)$ and dividing it by $\Delta t$, we have

$$
a_{0}{ }^{k}-\frac{a_{0}{ }^{k-1}}{\Delta t}=\frac{s_{n}{ }^{2}(k \Delta t)}{n^{2} \Delta t} \alpha_{k} c_{0}{ }^{k},
$$

the left hand side of which is uniformly bounded by Assumption $E$. As to the right hand side we have from Assumption $D$ and (4.5)

$$
\frac{s_{n}^{2}}{n^{2} \frac{(k \Delta t)}{\Delta t}} \alpha_{k} \geqq \frac{b^{2}}{l^{2}} \sigma,
$$

so that $c_{0}{ }^{k}$ is uniformly bounded:

$$
\left|c_{0}{ }^{k}\right| \leqq M_{1}
$$

Putting $j=n$ in $(4 \cdot 1)$ and dividing it by $h_{n}(k \Delta t)$, we have

$$
\left(\alpha_{k}-n \beta_{k}\right) \frac{a_{n-1}^{k}}{h_{n}(k \Delta t)}+\left(\alpha_{k}+n \beta_{k}\right) \frac{a_{n+1}^{k}}{h_{n}(k \Delta t)}=0
$$

since $\left.a_{n}{ }^{k}=a_{n}{ }^{k-1}=0 . \quad a_{n-1}^{k} / h_{n}(k\lrcorner t\right)$ is uniformly bounded from $(4 \cdot 2)$, and hence we see that $\left.a_{n+1}^{k} / h_{n}(k\lrcorner t\right)$ is also uniformly bounded from $b^{2} \lambda_{b} / l^{2}$ $\leqq \alpha_{k}+n \beta_{k}$ and $\alpha_{k}-n \beta_{k} \leqq \alpha_{k}-\beta_{k} / 3 \leqq \lambda_{b}$. If we put $j=n$ again in (4.1) 
and divide it by $h_{n}{ }^{2}(k \Delta t)$, we have

$$
\alpha_{k} c_{n}{ }^{k}-\frac{n \beta_{k}}{h_{n}(k \Delta t)}\left\{\frac{a_{n-1}^{k}}{h_{n}(k \Delta t)}-\frac{a_{n+1}^{k}}{h_{n}(k \Delta t)}\right\}=0
$$

which shows, by $n \beta_{k} /\left\{\alpha_{k} h_{n}(k \Delta t)\right\} \leqq \kappa A l^{2} /\left(2 \sigma b^{2}\right)$, that $c_{n}{ }^{k}$ is also uniformly bounded:

$$
\left|c_{n}{ }^{k}\right| \leqq M_{2}
$$

Furthermore, from Assumption $E$, we have

$$
\max _{1 \leqq j \leqq n-1}\left|c_{j}{ }^{0}\right| \leqq M_{3}
$$

Now if we compute the identity

$$
\left\{P_{L}(k, j-1) a_{j-1}^{k}-2 P_{L}(k, j) a_{j}{ }^{k}+P_{L}(k, j+1) a_{j+1}^{k}\right\} / h_{n}{ }^{2}(k \Delta t)=0
$$

using $(4 \cdot 1)$, we obtain the following scheme similar to $(4 \cdot 1)$ satisfied by $\left\{c_{j}^{k}\right\}$ :

$$
\begin{gathered}
(4 \cdot 38)-\left\{\alpha_{k}-(j-1) \beta_{k}\right\} c_{j-1}^{k}+\left\{1+2\left(\alpha_{k}-\beta_{k}\right)\right\} c_{j}^{k}-\left\{\alpha_{k}+(j+1) \beta_{k}\right\} c_{j+1}^{k} \\
=\left(1-2 \beta_{k}\right)^{2} c_{j}^{k-1}, j=1,2, \cdots, n-1 ; k=1,2, \cdots, m .
\end{gathered}
$$

Let

$$
c_{M}{ }^{k}=\max _{0 \leqq j \leqq n}\left|c_{j}{ }^{k}\right|, \quad k=1,2, \cdots, m
$$

If $c_{M}{ }^{k} \neq\left|c_{0}{ }^{k}\right|,\left|c_{n}{ }^{k}\right|$, we have

$$
c_{M}{ }^{k} \leqq \frac{\left(1-2 \beta_{k}\right)^{2}}{1-4 \beta_{k}} c_{M}^{k-1}
$$

from (4.38) under Assumption $A, B$ and $C$. For sufficiently small $\Delta t$, there exists $\mu>0$ such that

$$
\frac{\left(1-2 \beta_{k}\right)^{2}}{1-4 \beta_{k}} \leqq 1+\mu \Delta t
$$

Hence, if we take into account the case where $\left|c_{j}{ }^{k}\right|$ attains the maximum at $j=0$ or $j=n$, we have

$$
\begin{aligned}
{c_{M}}^{k} & \leqq \max \left(\left|c_{0}{ }^{k}\right|,\left|c_{n}{ }^{k}\right|,(1+\mu \Delta t){c_{M}}^{k-1}\right) \\
& \leqq(1+\mu \Delta t) \max \left(\left|c_{0}{ }^{k}\right|,\left|c_{n}{ }^{k}\right|, c_{M}{ }^{k-1}\right) \\
& \leqq e^{\mu \Delta t} \max \left(\left|c_{0}{ }^{k}\right|,\left|c_{n}{ }^{k}\right|, c_{M}{ }^{k-1}\right) .
\end{aligned}
$$


Putting $t=k \Delta t$, we conclude from $(4 \cdot 34),(4 \cdot 35)$ and $(4 \cdot 36)$ that

$$
\left|c_{j}{ }^{k}\right| \leqq e^{\mu t} \max \left(M_{1}, M_{2}, M_{3}\right) \leqq e^{\mu T} \max \left(M_{1}, M_{2}, M_{3}\right) \text {. }
$$

Q.E.D.

Lemma 6 (lumped mass system with $\theta=1$ ). Under Assumptions $A, B, C$ and $E$,

$$
\left|z_{n}(t)-z_{n}(t-\Delta t)\right| \leqq M_{4} \Delta t^{1 / 2}
$$

Proof. Putting $j=n-1$ in $(4 \cdot 1)$ and dividing it by $\Delta t$, we have

$$
\alpha_{k} \frac{h_{n}{ }^{2}(k \Delta t)}{\Delta t} c_{n-1}^{k}-\frac{(n-1) \beta_{k} a_{n-2}^{k}+a_{n-1}^{k}-a_{n-1}^{k-1}}{\Delta t}=0 \text {. }
$$

Since $c_{n-1}^{k}$ is uniformly bounded and

$$
\alpha_{k} \frac{h_{n}^{2}(k \Delta t)}{\Delta t} \leqq \frac{l^{2}}{b^{2}} \sigma+\frac{\kappa A \sigma l^{2}}{6 \lambda_{b} b^{3}} \Delta t
$$

the first term of the left hand side of $(4 \cdot 45)$ is uniformly bounded, so that

$$
\left|(n-1) \beta_{k} a_{n-2}^{k}+a_{n-1}^{k}-a_{n-1}^{k-1}\right| \leqq M \Delta t .
$$

Dividing the both sides by $h_{n}(k \Delta t)$, we have

$$
\begin{aligned}
& \mid 2(n-1) \beta_{k} \frac{a_{n-2}^{k}}{2 h_{n}(k \Delta t)}+\left\{\frac{a_{n-1}^{k}}{h_{n}(k \Delta t)}-\frac{a_{n-1}^{k-1}}{h_{n}((k-1) \Delta t)}\right\} \\
& \quad+a_{n-1}^{k-1}\left\{\frac{1}{h_{n}((k-1) \Delta t)}-\frac{1}{h_{n}(k \Delta t)}\right\} \leqq M \frac{\Delta t}{h_{n}(k \Delta t)} .
\end{aligned}
$$

If we put $j=2$ in $(3 \cdot 13)$ of Lemma 2 and use (4.4), we have

$$
\left|2(n-1) \beta_{k} \frac{a_{n-2}^{k}}{2 h_{n}(k \Delta t) \mid \leqq}\right| \leqq \frac{A^{2} \sqrt{\lambda_{b}}}{\sqrt{\sigma}} \Delta t^{1 / 2}
$$

From $(4 \cdot 3)$ we have

$$
\left|\frac{1}{h_{n}((k-1) \Delta t)}-\frac{1}{h_{n}(k \Delta t)}\right|=\left|\frac{2 n \beta_{k}}{\mid s_{n}(k \Delta t)-\Delta s_{n}(k \Delta t)}\right| \leqq \frac{\kappa A \sqrt{\lambda_{b}}}{\sqrt{\sigma b}} \Delta t^{1 / 2} .
$$

As to the right hand side of $(4 \cdot 47)$, we have an estimate

$$
\frac{\Delta t}{h_{n}(k \Delta t)}=\sqrt{\frac{n^{2} \Delta t}{s_{n}^{2}(k \Delta t)}} \Delta t^{1 / 2} \leqq \sqrt{\frac{\lambda_{b}}{\sigma}} \Delta t^{1 / 2}
$$


so that for sufficiently large $n$ we obtain from (4.47)

$$
\left|\frac{a_{n-1}^{k}}{h_{n}(k \Delta t)}-\frac{a_{n-1}^{k-1}}{h_{n}((k-1) \Delta t)}\right| \leqq M^{\prime} \Delta t^{1 / 2}
$$

This inequality in combination with the definition (4.6) gives (4.44).

Q.E.D.

It is easy to see from Lemma 5 and from the manner in which we extended $u_{n}(x, t)$ from $t=k \Delta t$ to intermediate values of $t$ that

$$
\lim _{x \rightarrow s_{n}(t)} \frac{\partial}{\partial x} u_{n}(x, t) \text { and } \lim _{x \rightarrow s_{\infty}(t)} \frac{\partial}{\partial x} u(x, t)
$$

exist (cf. Lemma 1 of [2]). Furthermore from (4.6)

$$
\text { (4.49) } \quad \lim _{x \rightarrow s_{n}(t)}-\kappa \frac{\partial u_{n}}{\partial t}(x, t)=z_{n}(t) \quad \text { (uniformly in } 0<t \leqq T \text { ) }
$$

so that we have

(4.50) $\lim _{n \rightarrow \infty} z_{n}(t)=-\kappa \frac{\partial u}{\partial x}\left(s_{\infty}(t), t\right) \quad$ (uniformly in $\left.0<t \leqq T\right)$.

By the definition $(4 \cdot 7)$, on the other hand, we have

$$
\begin{aligned}
s_{n}(t)=b+\kappa \frac{a_{n-1}^{0}}{h_{n}(0)} \Delta t & +\int_{\Delta t}^{t} z_{n}(\tau-\Delta t) d \tau \\
=b+\kappa \frac{a_{n-1}^{0}}{h_{n}(0)} \Delta t & +\int_{\Delta t}^{t} z_{n}(\tau) d \tau \\
& +\int_{\Delta t}^{t}\left\{z_{n}(\tau-\Delta t)-z_{n}(\tau)\right\} d \tau, \quad \Delta t \leqq t \leqq T .
\end{aligned}
$$

If we let $\Delta t \rightarrow 0$ in $(4.51)$, we have

$$
s_{\infty}(t)=b-\kappa \int_{0}^{t} \frac{\partial u}{\partial x}\left(s_{\infty}(\tau), \tau\right) d \tau
$$

from Lemma 6 and (4.50), and so we conclude

$$
\frac{d s_{\infty}(t)}{d t}=-\kappa \frac{\partial u}{\partial x}\left(s_{\infty}(t), t\right) .
$$

We proved the convergence of the scheme so far, but we also proved the existence of the solution of $(1 \cdot 1) \sim(1 \cdot 4)$ under Assumptions $A$ and $E$. 
Camnon and Hill [2] prored the uniqueness of the solution of $(1 \cdot 1)$ $\sim(1 \cdot 4)$, so that we have the following convergence

Theorem 2 (lumped mass system rcith $\theta=1$ ). Under Assumption $A, B, C, D$ and $E$, the approximate solution obtained by $(2 \cdot 23)$ $\sim(2 \cdot 26)$ converges to the unique solution of the Stefan problem (1.1) $\sim(1 \cdot 4)$ as $\lrcorner t \rightarrow 0 \quad(n \rightarrow \infty)$.

\section{§ 5. Numerical Example}

We shall show here a numerical result of the application of the present scheme to the following model problem:

$$
\begin{aligned}
& \frac{\partial u}{\partial t}=\frac{\partial^{2} u}{\partial x^{2}}, \quad 0<x<s(t), \quad 0<t \leqq 1 \\
& u(0, t)=g(t)=1-\frac{1}{2} t, \quad 0 \leqq t \leqq 1 \\
& u(x, 0)=f(x)=1-x, \quad b=s(0)=1 \\
& \frac{d s}{d t}=-u_{x}(s(t), t) .
\end{aligned}
$$

The actual computation was carried out using the lumped mass system with $\theta=1$. We employed $n=8,16,32,64$ and $m=4 n^{2}$, i.e. $\Delta t=1 /\left(4 n^{2}\right)$. In this example, $A=1, l=2, \lambda_{b}=1 / 16$ and $\lambda_{l}=1 / 4$, so that all Assumptions $A, B, C, D$ and $E$ are satisfied except $\frac{d g}{d t}(0)=\frac{d^{2} f}{d x^{2}}(0)$. Fig. 5 represents the solution $u_{n}(x, t)$ for $n=16, m=1024$, and Fig. 6 represents $s_{n}(t)$.

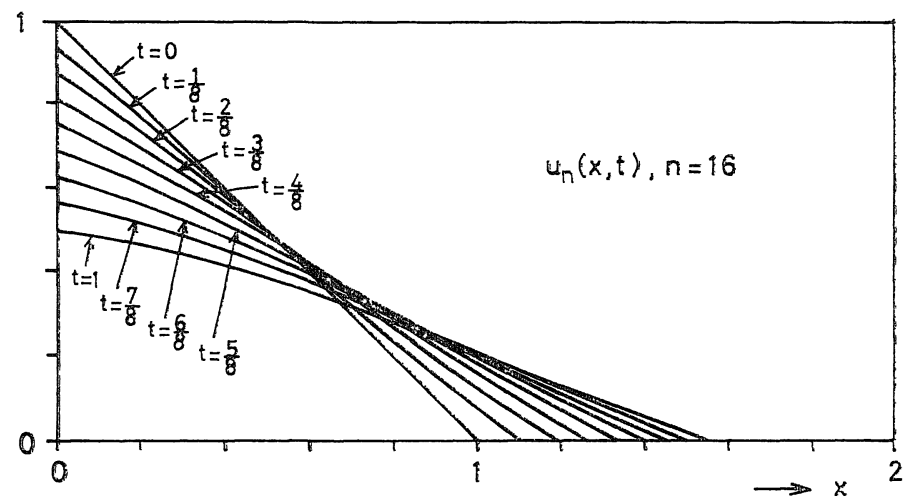

Fig. 5. Approximate solution $\iota_{n}(x, t)$. 


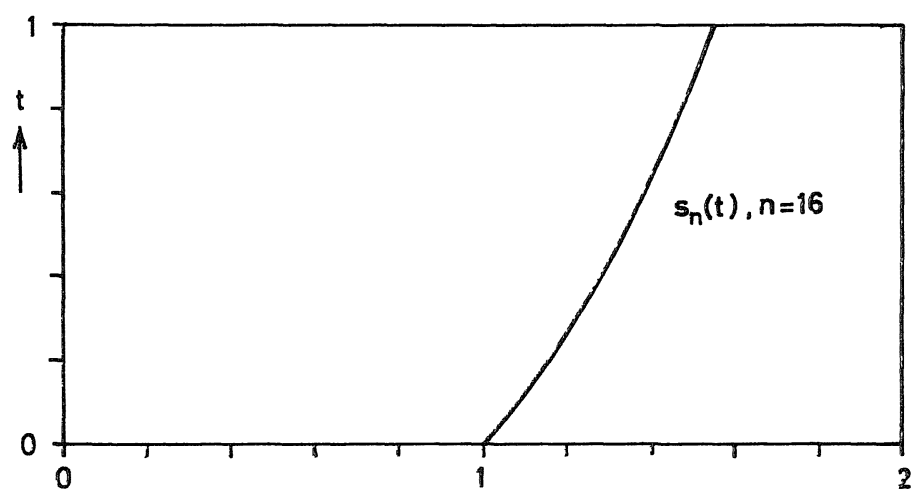

Fig. 6. The change of the free boundary $s_{n}(t)$.

In order to see the speed of convergence, we show the differences between the results for $n=8,16,32$ and that for $n=64$ in Table 1 . Both the rate of convergence of $s_{n}(t)$ and that of $u_{n}(x, t)$ seem to be approximately of the order of $1 / n$.

Table 1. The rate of convergence. $u_{n}(j, 1)$ and $u_{64}(j, 1)$ are the values at $x$ $=\frac{j}{n} S_{n}(1)$ and $t=1$, and $s t$ is equal to $1 / m$.

\begin{tabular}{r|r|r|r}
\multicolumn{1}{c|}{$n$} & \multicolumn{1}{c}{$m$} & $\max _{0 \leqq j \leqq n}\left|u_{n}(j, 1)-\iota_{64}(j, 1)\right|$ & $\max _{0 \leqq k \leqq m}\left|s_{n}(k \Delta t)-s_{64}(k \Delta t)\right|$ \\
\hline 8 & 256 & $3.34 \times 10^{-4}$ & $15.4 \times 10^{-3}$ \\
16 & 1024 & $1.55 \times 10^{-4}$ & $6.41 \times 10^{-3}$ \\
32 & 4096 & $0.539 \times 10^{-4}$ & $2.11 \times 10^{-3}$
\end{tabular}

Finally we note that the present idea is easy to apply to the two phase problem or to problems in higher space dimension [8].

\section{References}

[1] Bonnerot, R. and Jamet, P., A second order finite element method for the onedimensional Stefan problem, International J.for Numerical Methods in Engineering, 8 (1974), 811-820.

[2] Cannon, J. R. and Hill, C. D., Exsistence, uniqueness, stability, and monotone dependence in a Stefan problem for the heat equation, J. Math. and Mech., 17 (1967), 1-19.

[3] Douglas, J. Jr. and Gallie, T. M., On the numerical integration of a parabolic differential equation subject to a moving boundary condition, Duke Math. J., 22 (1955), 557-571.

[4] Fujii, H., Some remarks on finite element analysis of time-dependent field problems, Theory and Practice in Finite Element Structural Analysis, eds. Y. Yamada and 
R. H. Gallagher, University of Tokyo Press, 1973, 91-106.

[5] Kawarada, H. and Natori, M., On numerical solution Stefan problem I. Memoirs of Numerical Mathematics, No. 1 (1974), 43-54.

[6] Landau, H. G., Heat conduction in a melting solid, Quart. Appl. Math., 8 (1950), 81-94.

[7] Mori, M., Numerical solution of the Stefan problem by the finite element method. Memoirs of Numerical Mathematics, No. 2) (1975), 35-44.

[8] - - A finite element method for solving moving boundary problems, Preprints of IFIP Working Conference on Modelling of Environmental Systems, Tokyo, April 1976, 167-171.

[9] Nogi, T., A difference scheme for solving the Stefan problem. Publ. RIMS, Kyoto Univ., 9 (1974), 543-575.

[10] Petrovski, I. G., Partial Differential Equations, Iliffe Books, 1967 (translated from Russian ed.), Supplement. 
